\title{
A new way to target p53-defective colorectal cancer
}
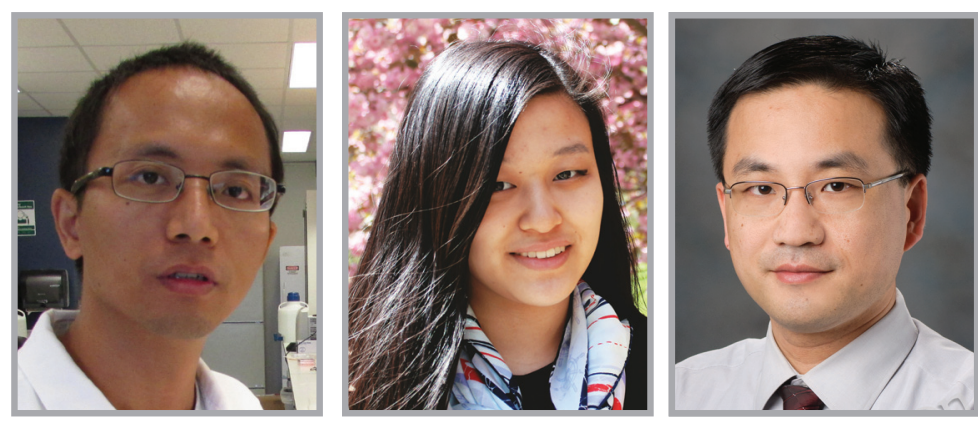

Yunhua Liu', Liana Wang' \& Xiongbin Lu ${ }^{*}, 1$

In the USA, colorectal cancer (CRC) is the fourth most common type of cancer in men and women and the second leading cause of cancer-related death, leading to the loss of nearly 50,000 patients per year. Deaths from colorectal cancer have decreased with the use of colonoscopy and fecal occult blood test. However, we have made little progress in treating patients with metastatic CRC despite advances in understanding the molecular genetics of this disease. The median overall survival for patients with metastatic CRC has only modestly increased to 31 months in the past few decades [1]. Our current drug development paradigm has failed. For example, the addition of monoclonal antibodies against EGFR to the backbone of multiagent chemotherapy has led to either no or mild benefit in both overall and progression-free survival $[2,3]$.

\section{Colorectal cancer genomics \& p53 deficiency}

Colorectal tumor imitation and progression are associated with acquired genomic events including genetic and epigenetic abnormalities. Chromosomal instability occurs in a majority of CRCs while
20-30\% of CRCs display characteristic features of $\mathrm{CpG}$ island methylator phenotype and microsatellite instability $[4,5]$. Comprehensive analysis of CRC genomics has identified significantly mutated genes, which include APC, TP53, SMAD4, PIK3CA, KRAS, ARIDIA, SOX9 and FAM123B mutations. The number of somatic mutations contributing to the pathogenesis of CRC is likely to be far greater than previously thought, and each individual tumor exhibits a distinct mutational gene signature. The tumor suppressor p53, encoded by the TP53 gene, is a central player in regulating cellular and organismal activities that span from cell proliferation and apoptosis to development, genome surveillance and aging [6]. TP53 is one of the most frequently mutated tumor suppressor genes in human cancers including CRC. Approximately half of human cancers harbor TP53 mutations, including genomic deletion, chromosomal translocation and single base substitutions. Moreover, increased levels of p53-negative regulators, such as $\mathrm{Mdm} 2$ and $\mathrm{Mdmx}$, downregulate $\mathrm{p} 53$ function in many of the other half of human cancers [6]. The universal loss of $\mathrm{p} 53$ activity in tumors has

'Department of Cancer Biology, The University of Texas MD Anderson Cancer Center, Houston, TX, USA

*Author for correspondence: Tel.: +1 713795 6247; Fax: +1 713563 5489; xlu2@mdanderson.org

\section{KEYWORDS}

- $\alpha$-amanitin • colorectal cancer

- hemizygous loss • p53 • POLR2A

- tumor suppressor gene

"In the USA, colorectal cancer is the fourth most common type of cancer in men and women and the second leading cause of cancer-related death, leading to the loss of nearly 50,000 patients per year.” 
“The principle of collateral vulnerability opens a door for the development of novel cancer therapeutics.” spurred tremendous efforts to develop new cancer treatments aimed at restoring the activity of p53. As examples, gene therapy using adenoviral vectors expressing wild-type $\mathrm{p} 53$ has shown activity in several clinical trials [7], but variable and insufficient gene delivery to every cancer cell and the presence of antibodies to adenovirus in humans has limited its clinical application. A number of small chemical compounds that target p 53 inhibitors have also been developed, one of which is the Nutlin family of inhibitors that block the Mdm2-p53 or Mdmx-p53 interaction [8]. However, this strategy is only applicable to cancers expressing wild-type $\mathrm{p} 53$, not for those cancers with mutant or no $\mathrm{p} 53$ expression.

Unlike many proteins, the level of $\mathrm{p} 53$ protein in human cells is determined primarily by its post-translational modifications such as ubiquitination, phosphorylation and acetylation, but independent of mRNA transcript levels [7]. Correspondingly, our analysis of The Cancer Genome Atlas and Reverse Phase Protein Array databases revealed that there is almost no correlation between TP53 gene copy numbers and p53 protein expression levels [9]. This seemingly surprising result challenges those clinical strategies that simply translate cancer genomics data to the prognosis of $\mathrm{p} 53$-targeted therapies. Up to now, no p53-based therapy has been successfully translated into clinical cancer treatment due to the complexity of p53 signaling. Therefore, instead of restoring p53 activity, identifying vulnerabilities conferred by TP53 deletion is emerging as a novel strategy for fighting against p53 deficiency in cancer. Our analysis of human cancer genomes in The Cancer Genome Atlas revealed that hemizygous deletion of TP53 is a very frequent event in many types of human cancer including colorectal, breast, ovarian and pancreatic cancer [9]. Therefore, a therapeutic strategy that is specific to this type of deletion may have broad applications.

One of the major features of cancer genomes is gene copy variation caused by genome instability and DNA repair defects in cancer cells. In particular, oncogene amplification and tumor suppressor gene deletion are primary driver events during tumor initiation and progression. Focal deletion regions in cancer cells often include many of the neighboring genes adjacent to the key tumor suppressor gene. While these passenger deletions do not contribute to or promote tumorigenesis, the partial loss of such neighboring genes render cancer cells highly vulnerable to further suppression or inhibition of those genes [10]. A genome-wide screen by Nijhawan and colleagues identified cancer-specific vulnerabilities due to gene copy number losses [11]. Their systematic analyses of copy number variation and gene essentiality screened out 56 genes for which gene suppression profoundly inhibited the proliferation and survival of cells bearing hemizygous loss of that gene. However, the number of these candidate genes was most probably underestimated, as small hairpin (shRNA) library screens used in their study was limited to a large extent by the knockdown efficacy of individual shRNA. Rapid progress in the CRISPR-Cas9 RNA editing technology now provides an efficient and reliable way to make precise and targeted changes to genes of interest in living cells. This powerful tool enables the genome-wide identification of essential genes in human cells [12]. Once essential genes are identified in the deleted regions, the next task is to search for a druggable target for clinical intervention.

\section{Targeting POLR2A in colorectal cancer harboring hemizygous loss of TP53}

In human cancers containing hemizygous deletion of TP53, a number of essential genes are codeleted due to their proximity to the TP53 gene, from which we identified POLR2A as one of the most promising drug targets based on the essentiality, druggability and co-deletion frequency of each candidate gene. POLR2A encodes the largest and catalytic subunit RPB1 of 12 subunits in the RNA polymerase II ( $\mathrm{Pol}$ II) complex [13]. Because RNA Pol II is in charge of mRNA synthesis, a crucial and indispensable function for any living cell, complete knockout of $P O L R 2 A$ is lethal to any type of cell. As normal cells express abundant POLR2A proteins, hemizygous loss of TP53/POLR2A has minimal effects on cell proliferation and survival. However, this loss creates a therapeutic vulnerability in cancer cells. We found that suppression of POLR2A with its inhibitors $(\alpha$-amanitin, small interfering RNAs [siRNAs] or shRNAs) selectively inhibits proliferation, survival and tumorigenic potential of colorectal cancer cells with hemizygous TP53 loss, suggesting a novel and effective strategy for cancers with such genomic alterations. $\alpha$-amanitin is the main component of amatoxins, cyclic 8-aa peptides from several species of the Amanita genus of mushrooms. It binds RNA Pol II of eukaryotic cells with the highest affinity (dissociation constant $[\mathrm{KD}]$ of $\sim 10^{-9} \mathrm{M}$ ) found 
for mammalian enzymes [14]. Free $\alpha$-amanitin causes apoptosis and necrosis of hepatocytes by interacting with the hepatocyte-specific transporting protein OATP1B3 [15]. Liver toxicity represents a major challenge in the clinical development of $\alpha$-amanitin [16]. However, this interaction is disrupted when $\alpha$-amanitin is conjugated to proteins such as antibodies. The $\alpha$-amanitin-antibody conjugates are no longer substrates for OATP1B3 [16]. We chemically conjugated $\alpha$-amanitin to a molecular antibody against EpCAM, a cell surface marker enriched in adenocarcinomas, including colorectal cancer [17]. This approach using antibody-drug conjugates (ADCs) will simultaneously overcome two bottlenecks in developing POLR2A inhibitors in preclinical studies: systematic toxicity and targeting specificity. In addition to $\alpha$-amanitin or the associated ADCs, siRNAs can be used to knock down the expression of POLR2A. Mook and colleagues designed siRNAs that specifically target a single nucleotide polymorphism in POLR2A and showed genotype-specific inhibition of tumor growth in vivo [18]. We also used a nanoliposomal delivery platform for systemic delivery of POLR2A siRNAs [9]. Mice treated with POLR2A siRNAs had pronounced growth reduction of xenografted colorectal tumors harboring hemizygous loss of POLR $2 A$.

The major challenge to targeted therapy is the development of drug resistance. For example, cells are under significant selection pressure when they are treated with a kinase inhibitor due to the important function of the kinase in cell proliferation, metabolism and survival [19]. Alternative signaling pathways will be activated or induced to compensate for the loss of activity of this important kinase. By contrast, the function of POLR2A is essential for cell survival, therefore, no alternative enzymes or pathways can take the place of POLR2A in mRNA synthesis in any type of cells, including therapyresistant cells and cancer stem cells. We anticipate that $\alpha$-amanitin-based ADCs will be a novel strategy to sensitize cancer cells in chemotherapy and target therapy. However, possible resistance mechanisms in the $\alpha$-amanitin-treated cells may be associated with the remaining POLR2A allele or those genes that regulate POLR2A, which include gene amplification or mutation, transcriptional activation of the remaining POLR2A allele, post-transcriptional or post-translational upregulation of POLR2A [20].

\section{Conclusion}

The principle of collateral vulnerability opens a door for the development of novel cancer therapeutics. In addition to CRC, inhibiting POLR2A will be an effective therapeutic strategy that is also applicable to other types of human tumors possessing a high frequency of hemizygous loss of TP53, such as breast, ovarian or pancreatic cancer. It is anticipated that many other essential genes in the tumor suppressordeleted regions will be identified and validated as potential drug targets for cancer therapy.

\section{Financial \& competing interests disclosure}

The study is supported by grants to X Lu from the Center for Genetics and Genomics Pilot Project Award, MD Anderson Cancer Center, and from the NIH/NCI (CA185742). The authors have no other relevant affiliations or financial involvement with any organization or entity with a financial interest in or financial conflict with the subject matter or materials discussed in the manuscript apart from those disclosed.

No writing assistance was utilized in the production of this manuscript.

\section{References}

1 Loupakis F, Cremolini C, Masi G et al. Initial therapy with FOLFOXIRI and bevacizumab for metastatic colorectal cancer. N. Engl. J. Med. 371(17), 1609-1618 (2014).

2 Douillard JY, Siena S, Cassidy J et al. Randomized, Phase III trial of panitumumab with infusional fluorouracil, leucovorin, and oxaliplatin (FOLFOX4) versus FOLFOX4 alone as first-line treatment in patients with previously untreated metastatic colorectal cancer: the PRIME study. J. Clin. Oncol. 28(31), 4697-4705 (2010).

3 Van Cutsem E, Kohne CH, Hitre E et al. Cetuximab and chemotherapy as initial treatment for metastatic colorectal cancer. N. Engl. J. Med. 360(14), 1408-1417 (2009).

4 Ogino S, Cantor M, Kawasaki T et al. CpG island methylator phenotype (CIMP) of colorectal cancer is best characterised by quantitative DNA methylation analysis and prospective cohort studies. Gut 55(7), 1000-1006 (2006).

5 Wood LD, Parsons DW, Jones S et al. The genomic landscapes of human breast and colorectal cancers. Science 318(5853), 1108-1113 (2007).

6 Toledo F, Wahl GM. Regulating the p53 pathway: in vitro hypotheses, in vivo veritas. Nat. Rev. Cancer 6(12), 909-923 (2006).
7 Vousden KH, Prives C. Blinded by the light: the growing complexity of p53. Cell 137(3), 413-431 (2009).

8 Wade M, Li YC, Wahl GM. MDM2, MDMX and p 53 in oncogenesis and cancer therapy. Nat. Rev. Cancer 13(2), 83-96 (2013).

9 Liu Y, Zhang X, Han C et al. TP53 loss creates therapeutic vulnerability in colorectal cancer. Nature 520 (7549), 697-701 (2015).

10 Frei E 3rd. Gene deletion: a new target for cancer chemotherapy. Lancet 342 (8872), 662-664 (1993). 
EDITORIAL Liu, Wang \& Lu

11 Nijhawan D, Zack TI, Ren Y et al. Cancer vulnerabilities unveiled by genomic loss. Cell 150(4), 842-854 (2012).

12 Wang T, Wei JJ, Sabatini DM, Lander ES. Genetic screens in human cells using the CRISPR-Cas9 system. Science 343(6166), 80-84 (2014).

13 Sawadogo M, Sentenac A. RNA polymerase B (II) and general transcription factors. Annu. Rev. Biochem. 59, 711-754 (1990).

14 Faulstich H, Trischmann H, Wieland T, Wulf E. Ether derivatives of alpha-amanitin. Introduction of spacer moieties, lipophilic residues, and radioactive labels. Biochemistry 20 (22), 6498-6504 (1981).
15 Letschert K, Faulstich H, Keller D, Keppler D. Molecular characterization and inhibition of amanitin uptake into human hepatocytes. Toxicol. Sci. 91(1), 140-149 (2006).

16 Moldenhauer G, Salnikov AV, Luttgau S, Herr I, Anderl J, Faulstich H. Therapeutic potential of amanitin-conjugated antiepithelial cell adhesion molecule monoclonal antibody against pancreatic carcinoma. J. Natl Cancer Inst. 104(8), 622-634 (2012).

17 Chaudry MA, Sales K, Ruf P, Lindhofer H, Winslet MC. EpCAM an immunotherapeutic target for gastrointestinal malignancy: current experience and future challenges. $\mathrm{Br}$ J. Cancer 96(7), 1013-1019 (2007).

18 Mook OR, Baas F, De Wissel MB, Fluiter K. Allele-specific cancer cell killing in vitro and in vivo targeting a single-nucleotide polymorphism in POLR2A. Cancer Gene Ther. 16(6), 532-538 (2009).

19 Garraway LA, Janne PA. Circumventing cancer drug resistance in the era of personalized medicine. Cancer Discov. 2(3), 214-226 (2012).

20 Bradner JE. Cancer: an essential passenger with p53. Nature 520(7549), 626-627 (2015). 